CLINICAL RESEARCH ARTICLE

\title{
Cerebral oxygenation and blood flow in normal term infants at rest measured by a hybrid near-infrared device (BabyLux)
}

Bjørn Andresen ${ }^{1}$, Agnese De Carli ${ }^{2}$, Monica Fumagalli ${ }^{2,3}$, Martina Giovannella ${ }^{4}$, Turgut Durduran ${ }^{4}$, Udo Michael Weigel ${ }^{5}$, Davide Contini $^{6}$, Lorenzo Spinelli ${ }^{7}$, Alessandro Torricelli ${ }^{6,7}$ and Gorm Greisen (iD)

BACKGROUND: The BabyLux device is a prototype optical neuro-monitor of cerebral oxygenation and blood flow for neonatology integrating time-resolved reflectance spectroscopy and diffuse correlation spectroscopy.

METHODS: Here we report the variability of six consecutive $30 \mathrm{~s}$ measurements performed in 27 healthy term infants at rest. Poor data quality excluded four infants.

RESULTS: Mean cerebral oxygenation was $59.6 \pm 8.0 \%$, with intra-subject standard deviation of $3.4 \%$, that is, coefficient of variation (CV) of 5.7\%. The inter-subject CV was $13.5 \%$. Mean blood flow index was $2.7 \times 10^{-8} \pm 1.56 \times 10^{-8}$ ( $\mathrm{cm}^{2} / \mathrm{s}$ ), with intra-subject CV of $27 \%$ and inter-subject CV of 56\%. The variability in blood flow index was not reduced by the use of individual measures of tissue scattering, nor accompanied by a parallel variability in cerebral oxygenation.

CONCLUSION: The intra-subject variability for cerebral oxygenation variability was improved compared to spatially resolved spectroscopy devices, while for the blood flow index it was comparable to that of other modalities for estimating cerebral blood flow in newborn infants. Most importantly, the simultaneous measurement of oxygenation and flow allows for interpretation of the high inter-subject variability of cerebral blood flow as being due to error of measurement rather than to physiological instability.

Pediatric Research (2019) 86:515-521; https://doi.org/10.1038/s41390-019-0474-9

\section{INTRODUCTION}

Despite improved treatment strategies, neurodevelopmental impairment is still a frequent consequence of preterm birth. Immature regulatory mechanisms for the control of cerebral blood flow (CBF) to ensure adequate oxygen delivery is thought to be a considerable part of the etiology.

The BabyLux project aimed to develop a device to continuously monitor cerebral oxygenation and blood flow-and hence monitor oxygen metabolism-by near-infrared spectroscopy (NIRS), integrating time-resolved reflectance spectroscopy (TRS) ${ }^{2}$ with diffuse correlation spectroscopy $(D C S)^{3}$ in a single device operating continuously under clinical conditions, providing robust measurements with a relatively high temporal resolution that would be suitable for use in neonates. ${ }^{4}$ In newborns, the distance from skin to brain is $<5 \mathrm{~mm}$, so optical methods could be particularly useful in this population. ${ }^{5}$

Most clinically approved NIRS devices use spatially resolved spectroscopy. ${ }^{6}$ To achieve absolute values these devices assume average optical properties for the investigated tissue. Such assumptions are possible sources of error, ${ }^{7}$ as optical properties reflect tissue composition, which changes during development of the central nervous system, for example, due to myelination. TRS measures the attenuation and temporal broadening as well as the time-of-flight of short light pulses propagating through tissue. By fitting measurements to a solution of the photon diffusion equation, TRS can separate absorption and scattering coefficients and resolve photon path lengths, allowing for direct, absolute determination of hemoglobin species concentrations. ${ }^{2}$

DCS relies on the interaction between long coherence laser light and moving scatters, which in tissue are red blood cells. The more moving scattering elements and the faster they move, the faster the loss of correlation of the reflected light is with its own source. Thus, an autocorrelation function can be used to quantify the microvascular blood flow in the illuminated tissue, and a blood flow index (BFI) can be calculated. ${ }^{3}$ It must be noted that optical properties, here measured by TRS, are needed as input parameters in order to retrieve absolute values of BFI. The combination of DCS and TRS allows for the assessment of an index of cerebral oxygen metabolism as tissue blood flow and oxygenation are measured simultaneously and independently.

The precision of quantitative measurements is a key parameter when evaluating the performance of medical devices, as clinical application usually targets normal ranges. The poorer the precision, the larger the confidence interval around a measurement becomes, and while this may be acceptable for research use, where the mean value for a number of subjects is relevant, poor precision always subtracts from the value for clinical decision making on the individual level. Clinically approved spatially resolved NIRS devices have usually been reported to have an intra-subject CV (standard deviation divided by the mean) of $6-7 \%$ on the newborn head ${ }^{8,9}$ determined by repeated repositioning of the probe.

\footnotetext{
'Department of Neonatology, Rigshospitalet and Copenhagen University, Copenhagen, Denmark; ${ }^{2}$ NICU Fondazione IRCCS Ca' Granda Ospedale Maggiore Policlinico, Milan, Italy;

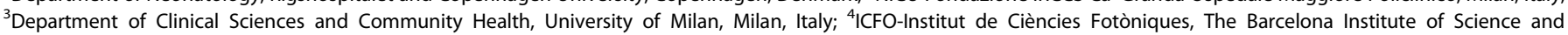

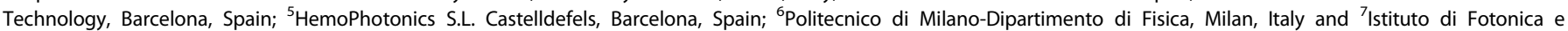
Nanotecnologie, Consiglio Nazionale delle Ricerche, Milan, Italy

Correspondence: Gorm Greisen (gorm.greisen@regionh.dk)
}

Received: 11 March 2019 Revised: 17 May 2019 Accepted: 14 June 2019

Published online: 24 June 2019 
In this paper, we report on the precision of the measurements taken with the BabyLux device in the clinical setting of healthy, term newborns at rest during their second day of life. A predefined goal of the BabyLux project was to improve the precision compared to spatially resolved NIRS devices.

\section{MATERIALS AND METHODS}

\section{Protocol}

The protocol was approved by local research ethics committees and national device agencies in both Italy and Denmark, and written informed consent was obtained from parents or legal guardian prior to inclusion. The clinical investigation has been monitored by external consultants according to ISO 14155:2011 "Clinical investigation of medical devices for human subjectsGood clinical practice." The protocol is registered at ClinicalTrials. gov, identifier NCT02815618. The repeatability of measurements was evaluated by reapplying the BabyLux probe on the frontoparietal region with the infant at rest. We targeted roughly the same position of the probe for every measurement, but did not use any markings on the skin to optimize precise repositioning and it is reasonable to expect variation in the positions to be $<5$ $\mathrm{mm}$. A total of six measurement runs of $30 \mathrm{~s}$ duration each were completed for each infant with a total lift-off of the probe in between-the measurement-to-measurement interval normally lasting $<60 \mathrm{~s}$. Within each measurement run the acquisition time was $1 \mathrm{~s}$ for both DCS and TRS, thus a total of 180 measurements were obtained from six slightly varying positions within the same region for each infant. During measurements the probe was kept in place by a self-adhesive elastic bandage, and ambient light reduced by dark cloth, if needed, in case the instrument reported an unacceptable level of background light.

\section{Subjects}

Measurements were performed during the second day of life. Twenty-seven term infants delivered by elective cesarean section with a median (range) gestational age of $38+3(37+0-9+5)$ weeks and birth weight of $3248(2420-4330) \mathrm{g}$ were included. Fourteen infants were enrolled in Milan, Italy and 13 in Copenhagen, Denmark. All infants were delivered without complications and had full Apgar scores at $10 \mathrm{~min}$ of age. Exclusion criteria were need for resuscitation or supplementary oxygen during the first 10 min following umbilical cord clamping, and congenital malformations expected to influence cerebral hemodynamics or oxygenation.

\section{Instrumentation}

The BabyLux device integrates TRS and DCS modules similar to those previously described by Torricelli et al. ${ }^{2}$ and Durduran and Yodh, ${ }^{3}$ respectively. In brief, the TRS module employs three pulsed lasers at 685,760 , and $820 \mathrm{~nm}$. The pulse duration is $<100 \mathrm{ps}$, with a repetition rate of $20 \mathrm{MHz}$, at an average output power $<1 \mathrm{~mW}$. A pulse driver enables proper delays of the three wavelengths allowing simultaneous acquisition within a $50 \mathrm{~ns}$ interval. TRS laser output is split by a fiber optic fused splitter in a reference branch, attenuated and fed directly to the photomultiplier, and a signal branch is directed to the probe to be placed on the tissue. Photons are collected by a multimode graded index plastic optical fiber in the probe, fed to the photomultiplier, and the distribution of time-of-flight (DTOF) for each wavelength accumulated in histograms. The DCS module uses a continuous wave long coherence laser at $785 \mathrm{~nm}$ carried in a multimode fiber with an output power of $20 \mathrm{~mW}$ at the probe surface regulated by a variable attenuator. DCS light is on for $9 \mathrm{~s}$ and off for $1 \mathrm{~s}$, in order to allow dissipation of heat. According to the standard UNEEN60825-1:2007, the maximum permissible exposure at $785 \mathrm{~nm}$ and with such exposure time is above the $20 \mathrm{~mW}$ value here used. The diffusely reflected light is collected in another window in the

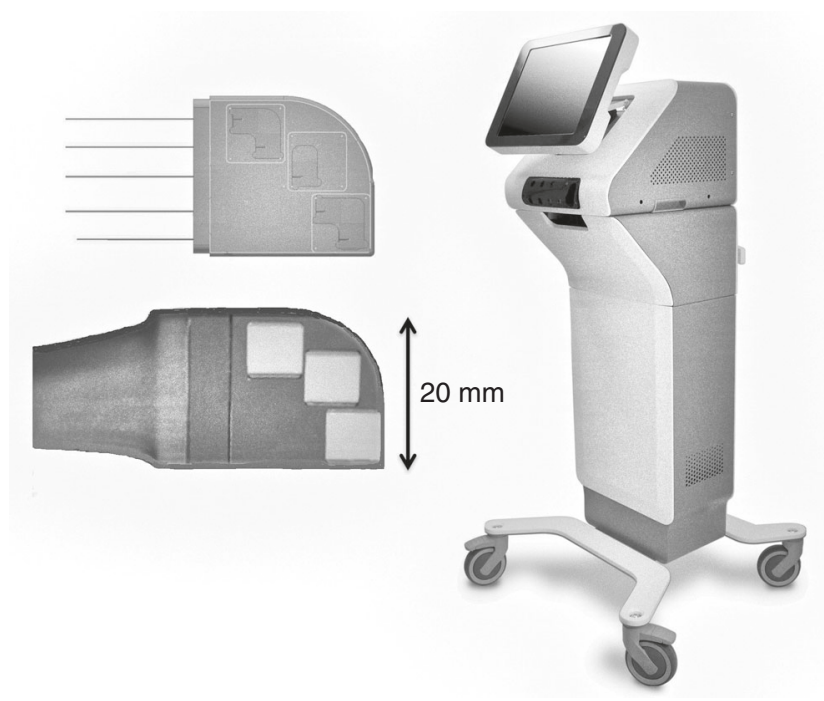

Fig. 1 The BabyLux device. To the right, the probe design with the entry of optical fibers and $15 \mathrm{~mm}$ source-detector distance and the probe surface with three sapphire windows. To the left, the cart configuration working as a stand-alone mobile device to be used at cot side or in the operating room

probe and measured by two single avalanche photodiodes. A custom-made correlator unit developed by HemoPhotonics S.L. computes the autocorrelation of the measured light intensity. The fiber optic probe head is made in aluminum with sapphire windows and deflects the light $90^{\circ}$ in order to have a compact design. TRS and DCS source-detector separation is $15 \mathrm{~mm}$. Since the light emission of the probe is not "eye-safe," a capacitive sensor designed to detect skin contact is integrated in the probe head in order to ensure safe operation of the device, as lasers can only be switched on if contact is detected. A detailed description of probe design is available in Rehberger et al. ${ }^{10}$ The prototype device was approved for research use according to the clinical investigation plan by the national Medical Device Agencies in both Italy and Denmark. Figure 1 shows the design of the BabyLux device and probe.

\section{Data processing and quality assessment}

The dedicated BabyLux software display real-time estimates of the measured tissue oxygenation $\left(\mathrm{S}_{\mathrm{t}} \mathrm{O}_{2}\right)$ and $\mathrm{BFI}$, and provide continuously feedback of the quality of the acquired data. However, data for the analysis for this report were postprocessed for thorough evaluation. The post-processing procedures of TRS data include estimation of the optical properties, determination of chromophore concentrations, and a quality evaluation of the results. For estimation of optical parameters (absorption coefficient $\left(\mu_{\mathrm{a}}\right)$ and reduced scattering coefficient $\left.\left(\mu_{\mathrm{s}}{ }^{\prime}\right)\right)$, the DTOF is fitted with a model for photon diffusion after convolution with the instrument response function. The semiinfinite homogeneous medium model for photon diffusion has been chosen in our case taking into account the optical and geometrical characteristics of neonates (i.e., limited thickness of scalp and skull and relatively large absorption), as it also enables depth sensitivity with less contribution from extracerebral layers using only one source-detector separation. ${ }^{11}$ The fitting procedure is described in detail in Cubeddu et al. ${ }^{12}$ Beer's law is used to determine the concentration of oxy-hemoglobin $\left(\mathrm{O}_{2} \mathrm{Hb}\right)$ and deoxy-hemoglobin $(\mathrm{HHb})$, from which total hemoglobin $(\mathrm{HbT}=$ $\left.\mathrm{O}_{2} \mathrm{Hb}+\mathrm{HHb}\right)$ and "tissue"-oxygenation, $\mathrm{S}_{\mathrm{t}} \mathrm{O}_{2}=\left(\mathrm{O}_{2} \mathrm{Hb}\right) / \mathrm{HbT}$ can be derived. Specific absorption values for hemoglobin are taken from the Prahl dataset. ${ }^{13}$ Lipid content in brain of neonates is limited and therefore disregarded, while water content is fixed at $90 \% .{ }^{14}$ 
TRS data quality is sensitive to the number of photons in the DTOF. We consider values of $>10^{3}$ photons per DTOF as acceptable. $X^{2}$ test is used to evaluate quality of the fit. Large values $\left(x^{2}>10\right)$ indicate a poor match between experimental data and fitting model and are discarded. Very low values $\left(x^{2}<0.1\right)$ are discarded as they may indicate a very low photon count. Further, the photon diffusion model assumes that photon loss due to scattering is much larger than absorption $\left(\mu_{\mathrm{s}}{ }^{\prime} » \mu_{\mathrm{a}}\right)$; hence, if the fit provides too low values for $\mu_{\mathrm{s}}^{\prime}$ (i.e., $<1 \mathrm{~cm}^{-1}$ ), the model may be violated, and values are excluded.

The normalized intensity field autocorrelation curve acquired in DCS measurements are fitted to the solution of the diffusion equation for the autocorrelation function for the semi-infinite homogeneous geometry. As the source-detector separation is known and optical properties at $760 \mathrm{~nm}$ have been estimated by TRS, they can be inserted in the model, enabling determination of BFI. DCS curves acquired with an intensity rate below $10 \mathrm{kHz}$ are excluded, due to poor signal-to-noise ratio, or when the laser is switching on or off for $1 \mathrm{~s}$ every $9 \mathrm{~s}$ (a safety precaution enabling measurements that last for hours). The optical properties influence $\mathrm{BFI}$, which is particularly sensitive to the estimate of $\mu_{\mathrm{s}}{ }^{\prime}$. In theory, $\mu_{\mathrm{s}}{ }^{\prime}$ is not expected to change considerably even during a change in blood flow. To balance the need for individual optical properties and measurement errors, BFI has been calculated with both the individual infant's $\mu_{\mathrm{s}}{ }^{\prime}$ averages and as well as the grand average of all individuals' $\mu_{\mathrm{s}}{ }^{\prime}$. In both cases data for $\mu_{\mathrm{s}}{ }^{\prime}$ at $760 \mathrm{~nm}$ were used. Since $\mu_{\mathrm{a}}$ varied less and is less critical for the calculation of BFI, only the individual averages were used. DCS results with residuals $>2$ SD from the individual mean have been rejected.

Statistical analysis

The BFI was logarithmically transformed (log(BFI)) to obtain a normal distribution before further analysis. One-way analysis of variance (ANOVA) with $\mu_{\mathrm{s}}{ }^{\prime}, \mu_{\mathrm{a}}, \mathrm{S}_{\mathrm{t}} \mathrm{O}_{2}, \mathrm{HbT}$, and $\log (\mathrm{BFI})$ as dependent variables and subject as random factor was used for the determination of within-subjects and between-subjects variance over the six replacements. Variation for variables following a normal distribution is reported as coefficient of variance (CV). BFI is reported as geometric statistics for log-normal distributions. The correlation between $\mathrm{S}_{\mathrm{t}} \mathrm{O}_{2}$ and $\log (\mathrm{BFI})$ were tested using Pearson's regression.

\section{RESULTS}

Of 27 enrolled infants, 23 were included in statistical analysis after applying quality criteria for the measurements as described above.
In 2 of the 23 infants, 1 of the $6 \mathrm{~S}_{\mathrm{t}} \mathrm{O}_{2}$ measurements did not meet the quality criteria, and in 7 of the 23 infants, 1 to 3 of the $6 \mathrm{BFI}$ measurements did not, 9 in total. Figures 2 and 3 show mean values of $\mathrm{S}_{\mathrm{t}} \mathrm{O}_{2}$ and $\mathrm{BFI}$ for each of the up to six $30 \mathrm{~s}$ measurements for all infants analyzed listed by subject. Figure 4 illustrates mean values of $\mu_{\mathrm{s}}{ }^{\prime}$ for each TRS wavelength over all measurements listed by subject.

The average $\mathrm{S}_{\mathrm{t}} \mathrm{O}_{2}$ value was $59.6 \pm 8.0 \%$ SD (range 37.0-72.5\%) across infants. The intra-subject standard deviation was $3.4 \%$, that is, the $\mathrm{CV}\left(\mathrm{CV}_{\text {intra }}\right)$ of $\mathrm{S}_{\mathrm{t}} \mathrm{O}_{2}$ was $5.7 \%$ and inter-subject $\mathrm{CV}\left(\mathrm{CV}_{\text {inter }}\right)$ $13.5 \%$. The BFI geometrical average was $2.71 \times 10^{-8} \mathrm{~cm}^{2} / \mathrm{s}$ (range $0.63 \times 10^{-8}-6.93 \times 10^{-8} \mathrm{~cm}^{2} / \mathrm{s}$ ) when using the grand average $\mu_{\mathrm{s}}{ }^{\prime}$ for all infants with $\mathrm{CV}_{\text {intra }}$ of $27 \%$ and $\mathrm{CV}_{\text {inter }}$ of $56 \%$. For $\mathrm{BFI}$ estimated using average $\mu_{\mathrm{s}}{ }^{\prime}$ for each individual infant geometric average BFI was $2.89 \times \times 10^{-8} \mathrm{~cm}^{2} / \mathrm{s}$ (range $0.89 \times 10^{-8}-2.57 \times 10^{-7}$ $\mathrm{cm}^{2} / \mathrm{s}$ ) with $\mathrm{CV}_{\text {intra }}$ of $28 \%$ and $\mathrm{CV}_{\text {inter }}$ of $89 \%$. Table 1 summarizes the main results for optical properties by wavelength and cerebral hemodynamics.

Tests for inhomogeneity of variance (Levene's test) were significant for both $\mathrm{S}_{\mathrm{t}} \mathrm{O}_{2}(p<0.001)$ and BFI $(p<0.0001)$ (Table 2), demonstrating that the replacement variability was particularly large in some infants as illustrated in Figs. 2 and 3.

The relationship between $\mathrm{S}_{\mathrm{t}} \mathrm{O}_{2}$ and $\log (\mathrm{BFI})$ did not show any correlation during the replacements $\left(R^{2}=0.003, p=0.524\right)$.

\section{DISCUSSION}

The absolute values of $\mathrm{S}_{\mathrm{t}} \mathrm{O}_{2}$ were comparable to previous reports using frequency domain NIRS, ${ }^{15,16}$ although several infants displayed low values (Fig. 2). It is noticeable that these values are not calibrated, but are modelled directly from in vitro extinction coefficients of oxy- and deoxy-hemoglobin. Spatially resolved NIRS devices have in general reported a higher $\mathrm{S}_{t} \mathrm{O}_{2}$. Studies of normal reference values for cerebral $\mathrm{S}_{\mathrm{t}} \mathrm{O}_{2}$ has focused on preterm or critically ill infants, but two studies report values around the second day of life in healthy term infants of $78 \pm 6-8 \%$ with the spatially resolved INVOS system. ${ }^{17,18}$ This is substantially higher than the $60 \pm 8 \%$ we observe. However, NIRS devices are known to vary greatly in absolute values and the INVOS with the neonatal/pediatric sensor measures notoriously higher values than most other systems, ${ }^{19}$ perhaps caused by the in vivo calibration that is often used.

Recently, using another TRS system (with $3 \mathrm{~cm}$ source-detector distance and 600 ps full-width at half-maximum of the instrument response function), TRS measurements on the head of 33 healthy

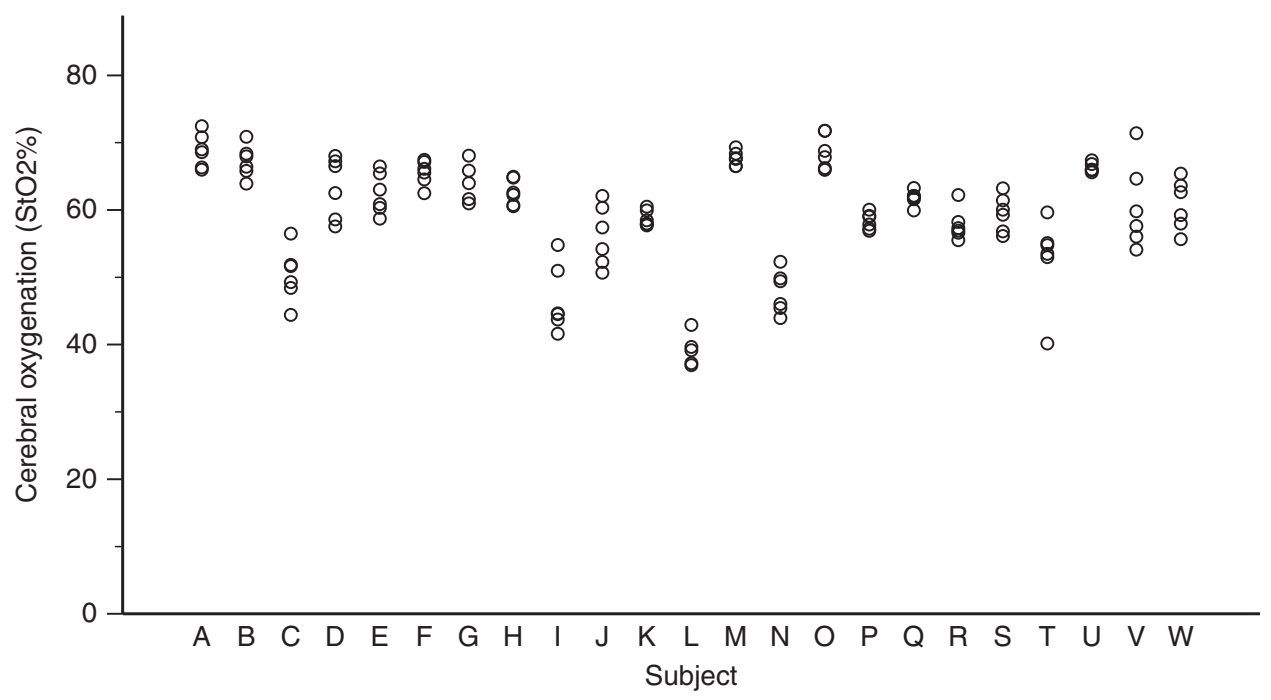

Fig. 2 Mean cerebral tissue oxygenation $\left(\mathrm{S}_{\mathrm{t}} \mathrm{O}_{2}\right)$ for up to six $30 \mathrm{~s}$ measurements in subjects $\mathrm{A}$ to $\mathrm{W}$ 


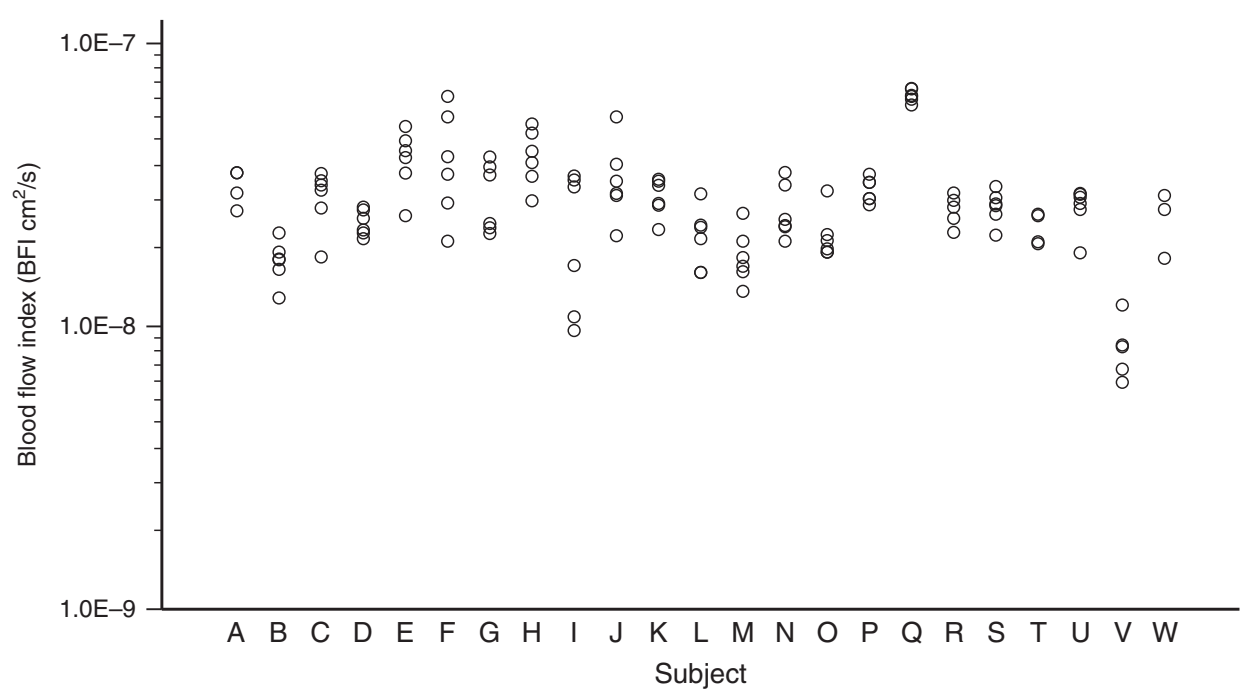

Fig. 3 Mean blood flow index (BFI) from each of the up to six $30 \mathrm{~s}$ measurements by in subjects $\mathrm{A}$ to $\mathrm{W}$ calculated using the grand average $\mu_{\mathrm{s}}{ }^{\prime}$

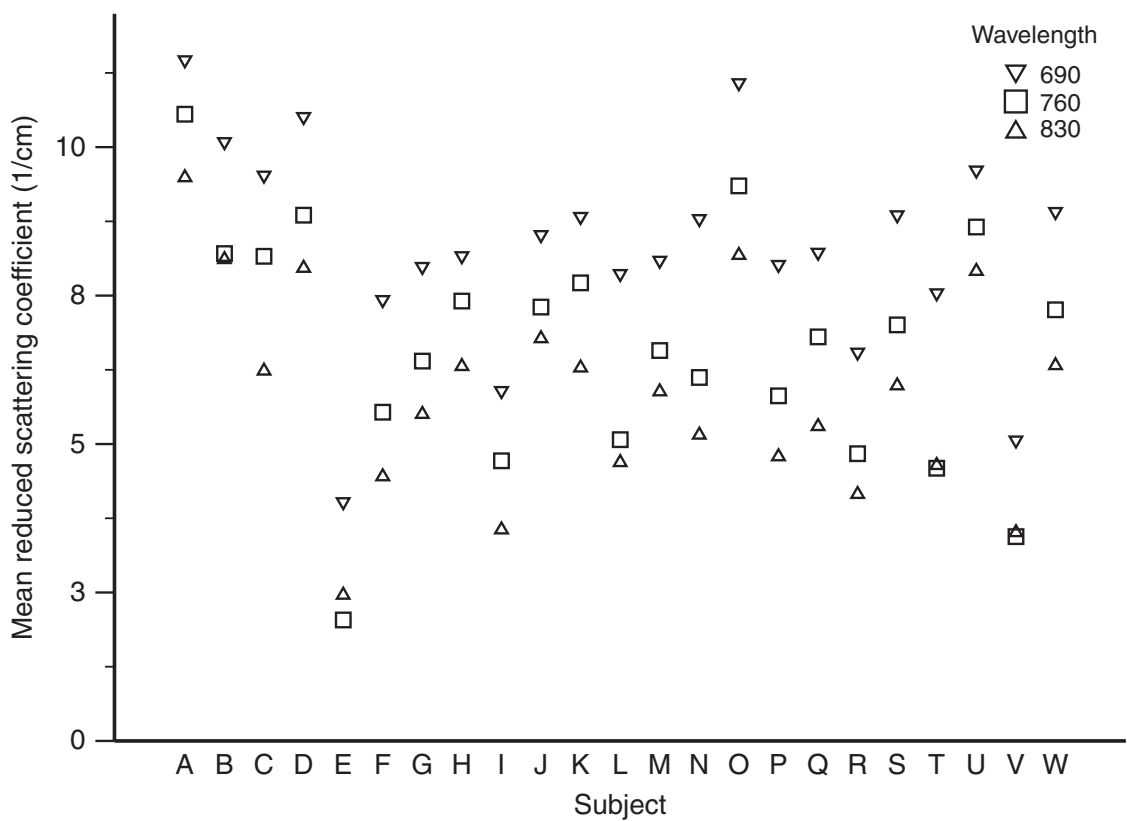

Fig. 4 Average individual values of $\mu_{s}{ }^{\prime}$ in subjects $A$ to $W$ by wavelength across all measurements

term infants around the third day of life were performed yielding a median $\mathrm{StO}_{2}$ of $72 \%$ and having approximately twice the absorption coefficient and total hemoglobin concentration than in our study. ${ }^{20}$ In that work, the combination of a TRS system with a significantly wider instrument response function with a larger source detector distance is likely to have resulted in a greater lateral spreading of photons, ${ }^{21}$ resulting in sampling from a larger volume of tissue (superficial and cerebral), as well as a greater effect of head curvature, that is, escape of photon head from boundaries, ${ }^{22}$ is likely to have contributed to the difference. Finally, the expected accuracy of the estimates of tissue optical properties $^{23,24}$ is lower than for the BabyLux system.

Absolute values of BFI were close to expected values, ${ }^{25}$ whereas the variability of the BFI appeared high, and was not reduced by use of individually measured $\mu_{s}^{\prime}$ as anticipated. Although BFI is an absolute quantification, the rather unconventional unit of $\mathrm{cm}^{2} / \mathrm{s}$ is not understandable for clinicians who are used to the unit $\mathrm{ml} / 100 \mathrm{~g} / \mathrm{min}$. Several studies have validated DCS against other modalities demonstrating a strong correlation, whereas only a few report calibrations. ${ }^{3}$ Using such a calibration, obtained in piglets comparing DCS with TRS combined with a flow tracer, ${ }^{26}$ our average BFI of $2.71 \times 10^{-8} \mathrm{~cm}^{2} / \mathrm{s}$ equals $23.6 \mathrm{ml} / 100 \mathrm{~g} / \mathrm{min}$, which is in the expected range of CBF for healthy term infants. ${ }^{27,28}$

Furthermore, neither the intra- nor the inter-subject variabilities were correlated between $\mathrm{S}_{\mathrm{t}} \mathrm{O}_{2}$ and $\mathrm{BFI}$. This could be expected, given that spontaneous fluctuations in $\mathrm{CBF}$ due to vasomotion would cause parallel changes in $\mathrm{S}_{\mathrm{t}} \mathrm{O}_{2}$, since it may be assumed that $\mathrm{CMRO}_{2}$ is relatively constant in healthy, term newborn infants at rest. These last findings suggest that the variabilities are for a significant part caused by measurement errors, rather than spontaneous changes in cerebral physiology during the periods the replacements were done.

It should be noted that no efforts were made to remove motion artifacts, besides the objective quality criteria stated above. Especially the BFI is sensitive to motion and to pressure applied by the bandage. This is also reflected in the large and 
Table 1. Main results of fitted and calculated variables

\begin{tabular}{|c|c|c|c|c|c|}
\hline & Mean & $S D_{\text {inter }}$ & Range & $\mathrm{CV}_{\text {inter }}(\%)$ & $\mathrm{CV}_{\text {intra }}(\%)$ \\
\hline$\mu_{\mathrm{a}(760)}\left(\mathrm{cm}^{-1}\right)$ & 0.185 & 0.038 & $0.106-0.263$ & 20 & 8 \\
\hline$\mu_{\mathrm{s}}^{\prime}(690)\left(\mathrm{cm}^{-1}\right)$ & 8.3 & 1.9 & $2.3-12.2$ & 23 & 9 \\
\hline$\mu_{\mathrm{s}}^{\prime}(760)\left(\mathrm{cm}^{-1}\right)$ & 6.6 & 2.1 & $1.1-11.3$ & 31 & 11 \\
\hline $\mathrm{HbT}(\mu \mathrm{M})$ & 75 & 20 & $31-120$ & 27 & 10 \\
\hline BFI (grand avr. $\left.\mu_{\mathrm{s}}^{\prime}\right)\left(\mathrm{cm}^{2} / \mathrm{s}\right)^{a}$ & $2.71 \times 10^{-8}$ & $1.56^{\mathrm{b}}$ & $0.63 \times 10^{-8}-6.93 \times 10^{-8}$ & 56 & 27 \\
\hline BFI $\left(\mu_{\mathrm{s}}^{\prime}\right.$ by subject avr.) $\left(\mathrm{cm}^{2} / \mathrm{s}\right)^{\mathrm{a}}$ & $2.89 \times 10^{-8}$ & $1.89^{\mathrm{b}}$ & $0.89 \times 10^{-8}-2.57 \times 10^{-7}$ & 89 & 28 \\
\hline
\end{tabular}

Table 2. Examples of commercially available near-infrared light-based devices to measure cerebral blood flow and cerebral oxygenation in newborn infants

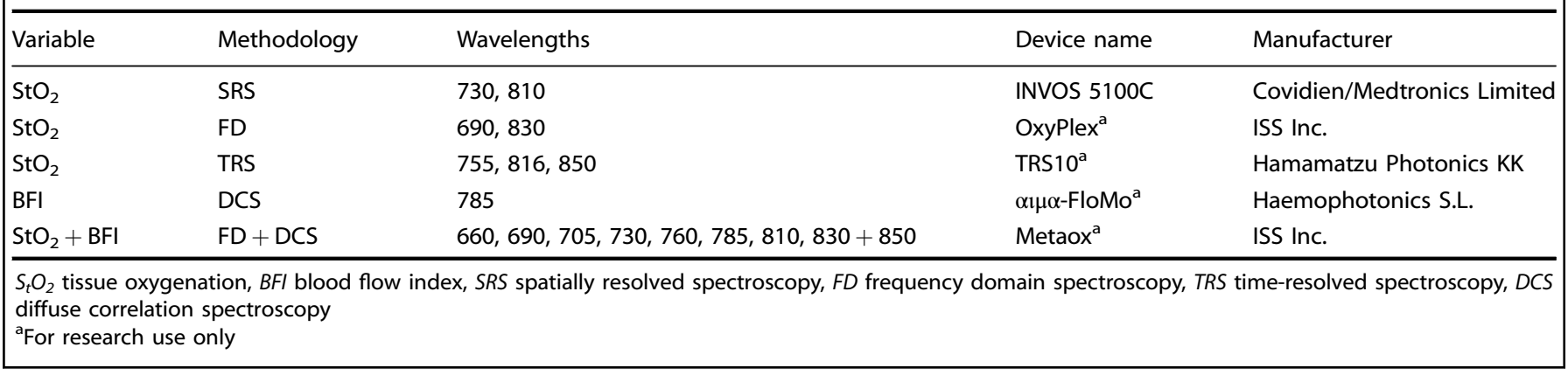

physiologically implausible range of CBF measurements, which spans a factor of more than 10 when calculated with the grand average $\mu_{\mathrm{s}}{ }^{\prime}$, and this variability was even larger when calculated with individual $\mu_{\mathrm{s}}^{\prime}$.

The assessment of variability between repositions in our protocol relies on the brain to be in a stable resting state throughout the measurement. This clinical test design aimed at evaluating "real-life" performance as expected under normal clinical conditions. The handling of the infant for the purpose of resiting the sensor with removal and re-application of the selfadhesive bandage may have resulted in some infants changing behavioral state. Further, normal physiological fluctuations relayed from systemic functions (e.g., arterial oxygen saturation, heart rate, respiration, and baro-reflex vasomotion) are reflected in cerebral hemodynamics and oxygenation despite autoregulation, or may even be an inherent trait of cerebral circulation improving its overall stability. ${ }^{29}$ In our protocol each measurement lasts $30 \mathrm{~s}$ $(0.033 \mathrm{~Hz})$. Since the sample rate will work as simple filter, intrasubject variability will not reflect variations caused by heart rate $(2-3 \mathrm{~Hz})$ and respiration (approx. $1 \mathrm{~Hz})$, but could vary with vasomotion $(0.1-0.01 \mathrm{~Hz})$ or arterial saturation (more random). However, the amplitudes of such physiologic oscillations in the cerebral hemodynamics are likely small compared to the variability observed in our study. ${ }^{30}$

The variation in CBF in the normal human newborn is not well known because of methodological limitations. Using the "goldstandard" method, positron emission tomography (PET) in the normal, resting adult, the $\mathrm{CV}$ is about $15 \%$, while after normalizing for differences in global $\mathrm{CBF}$, the $\mathrm{CV}$ in $\mathrm{CBF}$ is $5-10 \%$ among subjects and about $10 \%$ among cortical areas. ${ }^{32,33}$ In a recent study of healthy, resting newborn infants using PET, the $\mathrm{CV}$ of $\mathrm{CBF}$ in the frontal cortex among the four subjects was $23 \%{ }^{27}$ in good agreement with the adult situation. In preterm infants a similar $\mathrm{CV}_{\text {inter }}$ of $27 \%$ for CBF in the frontal region have been demonstrated using the ${ }^{133}$ Xenon clearance method. ${ }^{34}$ We therefore find it reasonable to assume that the true inter-subject variability is around $25 \%$ in normal, healthy term infants when transition after birth has taken place. This means that the BFI intersubject variability of $56 \%$ seen in the present study is increased by measurement error. A recent similar study using frequency domain NIRS and DCS had a comparable BFI inter-subject CV of $38 \%,{ }^{16}$ if ignoring the skewed nature of the data, which in our case would give a $\mathrm{CV}_{\text {inter }}$ of $43 \%$. However, it should be noted that measurement variability is not easily compared across modalities as temporal resolution varies greatly, from seconds with optical methods to minutes with PET, due to differences in protocol for data acquisition and processing. For comparison, the precision of the BFI obtained by the BabyLux instrument in a phantom and in a piglet model was in the order of $5 \%^{35}$ and the signal variability within the 30-s measurement periods in the dataset acquired in the present study was $10 \%$, only, as previously published. ${ }^{36}$

The BabyLux hybrid device used in the present study allowed simultaneous estimation of $\mu_{s}^{\prime}$ by TRS in each infant. When the DCS BFI was calculated using this individual $\mu_{\mathrm{s}}{ }^{\prime}$, the inter-subject $\mathrm{CV}$ was increased, not decreased. We interpret this as an indication that this "individualization" in effect added error to the estimation 
of CBF. Although the measurement of $\mu_{\mathrm{s}}{ }^{\prime}$ was ineffective in improving the precision of $\mathrm{BFI}$, the unexpectedly large intersubject variability of $\mu_{s}{ }^{\prime}$, suggests that one important reason for the very poor precision of BFI in human infants is optical heterogeneity, probably due to pools of cerebrospinal fluid due to relatively open sulci between the growing gyri. To support this, in four infants, the estimated $\mu_{\mathrm{s}}{ }^{\prime}$ for each of the wavelengths 690 , 760 , and $820 \mathrm{~nm}$, did not display the expected "linear" decrease (subject B, E, T, and V, Fig. 4). This was likely to be due to error of measurement, since the six replications were particularly variable in three of the four infants-possibly due to the marginally different average pathways of photons of different wavelength, and overall the deviation from the expected pattern in these four infants was hardly statistically significant.

Data on inter-subject variation of cerebro-venous oxygenation in normal newborn infants is also scarce. There are no data using blood sampling and co-oximetry due to ethical reasons. The first data was provided for seven infants using continuous wave NIRS and jugular venous occlusion. The inter-subject CV was $15.4 \%{ }^{37}$ Recently, magnetic resonance imaging was used to estimate oxygenation in the superior sagittal sinus in 10 infants, yielding a $\mathrm{CV}_{\text {inter }}$ of $16 \%$. Although brain $\mathrm{S}_{\mathrm{t}} \mathrm{O}_{2}$, estimated by TRS as in the present study, is a different quantity, the $\mathrm{CV}_{\text {inter }}$ was similar, $13.5 \%$, but apparently higher when estimated by frequency domain NIRS at $26 \%{ }^{16}$

The BabyLux device's improvement in intra-subject CV for $\mathrm{S}_{\mathrm{t}} \mathrm{O}_{2}$ seems modest, but should be assessed in the context of the lower mean value. Simply looking at the intra-subject standard deviation of $3.4 \%$, not affected by the mean values, the improvement is considerable, and corresponds to a reduction in the $95 \%$ confidence interval for a measurement to $\pm 9 \%$-points, as opposed to $\pm 13 \%$-points for spatial resolved NIRS. ${ }^{8,9}$ Recently, a new spatially resolved spectroscopy NIRS device has produced lower intra-subject CV for StO2, possibly related to interrogating a larger brain volume and/or an auto-calibration function. ${ }^{38}$ Reducing the confidence interval has a great impact on the clinical usefulness of a measurement as therapeutic ranges are often narrow and patient management often rely on intervention thresholds, making the reliability of the absolute value decisive.

\section{CONCLUSION}

We obtained simultaneous measurements of cerebral $\mathrm{S}_{\mathrm{t}} \mathrm{O}_{2}$ and $\mathrm{BFI}$ in healthy, term newborn infants at rest with acceptable short time reposition coefficient of variability of approximately $5 \%(\approx S D$ $3.4 \%$ ) and $25 \%$, respectively. The reposition variability of $\mathrm{S}_{\mathrm{t}} \mathrm{O}_{2}$ meets our predefined goal of improvement compared to spatial resolved NIRS devices, while the reposition variability of BFI is in the same range as other modalities for CBF measurement in infants, but with the advantage of being method that can be used bedside and in real time. For the inter-subject variability, the BabyLux device compared well with other modalities for cerebral $\mathrm{S}_{\mathrm{t}} \mathrm{O}_{2}-13.5 \%$ compared to typical values of $15 \%$, but not for CBF with $56 \%$ compared to typical values of $25 \%$. Most importantly, the simultaneous measure of oxygenation and flow, and the lack of correlation between these measures, allows to interpret the the high inter-subject variability of CBF as being due to error of measurement rather than physiological instability. Most likely true inter-individual variability of cerebral blood flow in healthy, stable, term infants is not greater than it is in other groups of healthy individuals.

\section{ACKNOWLEDGEMENTS}

We would like to thank all collaborators in the BabyLux consortium and the parents of the infants included in the clinical investigation. This research was funded by the European Commission Competitiveness for Innovation Program (grant agreement no. 620996) as part of the project titled: An optical neuro-monitor of cerebral oxygen metabolism and blood flow for neonatology (BabyLux).

\section{AUTHOR CONTRIBUTIONS}

Substantial contributions to conception, design, data acquisition. or analysis or interpretation: All. Drafting and revising article: B.A., A.D.C., M.F., M.G., D.C., L.S., A.T., G. G. Final approval: All.

\section{ADDITIONAL INFORMATION}

Competing interests: The IP generated during the BabyLux project is owned by the technological partners. U.M.W. is the CEO, has equity ownership in HemoPhotonics S. L., and is an employee in the company. His role in the project has been defined by the project objectives, tasks, and work-packages, and was reviewed by the European Commission. The other authors declare no competing interests.

Publisher's note: Springer Nature remains neutral with regard to jurisdictional claims in published maps and institutional affiliations.

\section{REFERENCES}

1. Volpe, J. J. Brain injury in premature infants: a complex amalgam of destructive and developmental disturbances. Lancet Neurol. 8, 110-124 (2009).

2. Torricelli, A. et al. Time domain functional NIRS imaging for human brain mapping. Neurolmage 85 Part 1, 28-50 (2014).

3. Durduran, T. \& Yodh, A. G. Diffuse correlation spectroscopy for non-invasive, microvascular cerebral blood flow measurement. Neurolmage 85 Part 1, 51-63 (2014).

4. Weigel, U. M. et al. The BabyLux project-an optical neuro-monitor of cerebral oxygen metabolism and blood flow for neonatology. Biomed. Opt. OSA Technical Digest (online), paper JM3A.30 (2016).

5. Greisen, G., Andresen, B., Plomgaard, A. M. \& Hyttel-Sørensen, S. Cerebral oximetry in preterm infants: an agenda for research with a clear clinical goal. Neurophotonics 3, 031407 (2016).

6. Scholkmann, F. et al. A review on continuous wave functional near-infrared spectroscopy and imaging instrumentation and methodology. Neurolmage 85 Part 1, 6-27 (2014).

7. Metz, A. J., Biallas, M., Jenny, C., Muehlemann, T. \& Wolf, M. The effect of basic assumptions on the tissue oxygen saturation value of near infrared spectroscopy. Adv. Exp. Med. Biol. 765, 169-175 (2013).

8. Hyttel-Sorensen, S., Hessel, T. W., la Cour, A. \& Greisen, G. A comparison between two NIRS oximeters (INVOS, OxyPrem) using measurement on the arm of adults and head of infants after caesarean section. Biomed. Opt. Express 5, 3671-3683 (2014).

9. Hessel, T. W., Hyttel-Sorensen, S. \& Greisen, G. Cerebral oxygenation after birth-a comparison of INVOS((R)) and FORE-SIGHT near-infrared spectroscopy oximeters. Acta Paediat. (Oslo, Norway: 1992) 103, 488-493 (2014).

10. Rehberger, M. et al. Fiber-based hybrid probe for non-invasive cerebral monitoring in neonatology. In Diffuse Optical Imaging V (H. Dehghani \& P. Taroni, eds.) Vol. 9538 of SPIE Proceedings (Optical Society of America, 2015), paper 95381J.

11. Giusto, A. et al. Monitoring absorption changes in a layered diffusive medium by white-light time-resolved reflectance spectroscopy. IEEE Trans. Instrum. Meas. 59, 1925-1932 (2010).

12. Cubeddu, R., Pifferi, A., Taroni, P., Torricelli, A. \& Valentini, G. Experimental test of theoretical models for time-resolved reflectance. Med. Phys. 23, 1625-1633 (1996).

13. Oregon Medical Laser Center. Optical Absorbption of Hemoglobin (Oregon Medical Laser Center, 1999). http://omlc.ogi.edu/spectra/hemoglobin.

14. Dobbing, J. \& Sands, J. Growth and development of human brain. Arch. Dis. Child 48, 757 (1973).

15. Demel, A. et al. Healthy term and moderately preterm infants have similar cerebral oxygen saturation and cerebral blood flow volumes during early postnatal transition. Acta Paediatr. 104, e330-336 (2015).

16. Farzam, P. et al. Shedding light on the neonatal brain: probing cerebral hemodynamics by diffuse optical spectroscopic methods. Sci. Rep. 7, 15786 (2017)

17. Bailey, S. M., Hendricks-Munoz, K. D. \& Mally, P. Cerebral, renal, and splanchnic tissue oxygen saturation values in healthy term newborns. Am. J. Perinatol. 31, 339-344 (2014).

18. Bernal, N. P., Hoffman, G. M., Ghanayem, N. S. \& Arca, M. J. Cerebral and somatic near-infrared spectroscopy in normal newborns. J. Pediatr. Surg. 45, 1306-1310 (2010).

19. Kleiser, S., Nasseri, N., Andresen, B., Greisen, G. \& Wolf, M. Comparison of tissue oximeters on a liquid phantom with adjustable optical properties. Biomed. Opt. Express 7, 2973-2992 (2016).

20. Spinelli, L. et al. In vivo measure of neonate brain optical properties and hemodynamic parameters by time-domain near-infrared spectroscopy. Neurophotonics 4, 041414 (2017). 
21. Feng, S., Zeng, F.-A. \& Chance, B. Photon migration in the presence of a single defect: a perturbation analysis. Appl. Opt. 34, 3826-3837 (1995).

22. Liemert, A. \& Kienle, A. Light diffusion in a turbid cylinder. I. Homogeneous case. Opt. Express 18, 9456-9473 (2010).

23. Wabnitz, H. et al. Performance assessment of time domain optical brain imagers, part 1: Basic physical performance protocol. J. Biomed. Opt. 19, 086011 (2014).

24. Zucchelli, L., Contini, D., Re, R., Torricilli, A. \& Spinelli, L. Method for the discrimination of superficial and deep absorption variation by time domain fNIRS. Biomed. Opt. Express 4, 2893-2910 (2013).

25. Lin, P. Y. et al. Regional and hemispheric asymmetries of cerebral hemodynamic and oxygen metabolism in newborns. Cereb. Cortex. (United States) 23, 339-348 (2013).

26. Giovannella, M. et al. Validation and Potential Calibration of Diffuse Correlation Spectroscopy versus (15)O-Water Positron Emission Tomography on Neonatal Piglets, fNIRS2018 (Society for Functional Near-infrared Spectroscopy, Tokyo, 2018).

27. Andersen, J. et al. Hybrid PET/MRI imaging in healthy unsedated newborn infants with quantitative rCBF measurements using (15)O-water PET. J. Cereb. Blood Flow Metab. 39, 782-793 (2019).

28. Greisen, G. Cerebral blood flow in preterm infants during the first week of life. Acta Paediatr. Scand. 75, 43-51 (1986).

29. Garrett, D. D. et al. Moment-to-moment brain signal variability: a next frontier in human brain mapping? Neurosci. Biobehav. Rev. 37, 610-624 (2013).

30. Kirilina, E. et al. Identifying and quantifying main components of physiological noise in functional near infrared spectroscopy on the prefrontal cortex. Front. Hum. Neurosci. 7, 864 (2013).
31. Henriksen, O. M. et al. Estimation of intersubject variability of cerebral blood flow measurements using MRI and positron emission tomography. J. Magn. Reson. Imaging 35, 1290-1299 (2012).

32. Vaishnavi, S. N. et al. Regional aerobic glycolysis in the human brain. Proc. Natl. Acad. Sci. USA 107, 17757-17762 (2010).

33. Aanerud, J., Borghammer, P., Rodell, A., Jonsdottir, K. Y. \& Gjedde, A. Sex differences of human cortical blood flow and energy metabolism. J. Cereb. Blood Flow. Metab. 37, 2433-2440 (2017).

34. Baenziger, O. et al. Regional differences of cerebral blood flow in the preterm infant. Eur. J. Pediatr. 154, 919-924 (1995).

35. Giovannella M. et al. Accuracy and precision of tissue optical properties and hemodynamic parameters estimated by the BabyLux device: a hybrid timeresolved near-infrared and diffuse correlation spectroscopy neuro-monitor Biomed. Opt. Express 10, 2556-2579 (2019).

36. Giovannella, M. et al. The BabyLux Device: Baseline Hemodynamic and Optica Properties of the Newborn Brain and the Reproducibility of Measurements. In Biophotonics Congress: Biomedical Optics Congress 2018 (Microscopy/Translational/ Brain/OTS), OSA Technical Digest (Optical Society of America, 2018), paper OW4C.2.

37. Buchvald, F. F., Kesje, K. \& Greisen, G. Measurement of cerebral oxyhaemoglobin saturation and jugular blood flow in term healthy newborn infants by nearinfrared spectroscopy and jugular venous occlusion. Biol. Neonate 75, 97-103 (1999).

38. Kleiser, S. et al. In vivo precision assessment of a near-infrared spectroscopybased tissue oximeter (OxyPremv1.3) in neonates considering systemic hemodynamic fluctuations. J. Biomed. Opt. 23, 067003 (2018).

39. Kirkwood, T. B. L. Geometric means and measures of dispersion. Biometrics 35 , 908-909 (1979). 